\title{
EMPOWER-support of patient empowerment by an intelligent self-management pathway for patients: study protocol
}

\author{
Sarah Mantwill, Maddalena Fiordelli, Ramona Ludolph and Peter J Schulz
}

\begin{abstract}
Background: Diabetes education together with patient empowerment has shown to be key to effective self-management behavior. When delivered through information and communication technologies (ICT), this solution has shown to lead to better health outcomes. However, the potential of ICT and their integration into the healthcare environment have not yet been fully exploited. ICT should be in particular used to facilitate communication and information exchange between patient and healthcare providers. In addition, systems should include components facilitating behavior change using empowerment approaches such as goal-setting.

Methods/Design: Funded by the European Commission (FP7-ICT-2011-288209) a web/mobile based platform (EMPOWER) has been developed, which aims at supporting self-management activities of diabetes patients and their treating physicians in Germany and Turkey. The platform semantically integrates multiple information sources, such as electronic and personal health records (EHR/PHR). Patients can register patterns of daily living, record blood glucose levels, design disease management plans and set long- and short-term goals. The project actively involves the treating physician, who has the possibility to set recommendations for the patient and to monitor his/her progress on the platform.

In the test-phase of EMPOWER, patients will be assigned to an intervention group and a control group. Data will be collected at baseline and three months after the intervention started. In addition, qualitative interviews will be conducted to collect extra information on usability and usefulness.

Outcome measures include amongst others the Problem Areas in Diabetes questionnaire (PAID), the Summary of Diabetes Self-Care Activities and scales evaluating doctor-patient interaction. Physiological parameters, such as physical activity or blood glucose levels will be collected via the platform. Further, log files and number of logins will serve as independent variables.
\end{abstract}

Discussion: The interplay between multiple sources, including EHR, patients' own registered information and physicians' recommendations on one platform can have important practice implications. It might not only improve self-management activities in diabetes patients but it will also facilitate physician's work, and ultimately the physician patient relationship.

Trial registration: The trial has been registered with Deutsches Register Klinischer Studien (German register of clinical trials) under DRKS00007699 on January 30, 2015.

Keywords: Empowerment, Self-management, Diabetes mellitus, mHealth, eHealth, ICT, Doctor patient communication, Study protocol

\footnotetext{
* Correspondence: sarah.mantwill@usi.ch

Institute of Communication \& Health, University of Lugano, Via Giuseppe Buffi 13, 6904 Lugano, Switzerland
}

\section{Biomed Central}

(c) 2015 Mantwill et al.; licensee BioMed Central. This is an Open Access article distributed under the terms of the Creative Commons Attribution License (http://creativecommons.org/licenses/by/4.0), which permits unrestricted use, distribution, and reproduction in any medium, provided the original work is properly credited. The Creative Commons Public Domain Dedication waiver (http://creativecommons.org/publicdomain/zero/1.0/) applies to the data made available in this article unless otherwise stated. 


\section{Background}

Diabetes, and in particular diabetes type 2, is one of the fastest growing chronic diseases in nowadays society [1]. Tight blood glucose control, dietary requirements and regular medication intake are only few of the things that a diabetes patient needs to manage in order to prevent long-term complications [2]. Given that continuity of care has to be guaranteed, this does not only pose many challenges for the patient but also for the treating physician who will need to support the patient's management strategies.

Research has shown that diabetes education is central to effective self-management behavior, which in the long term can influence clinical [3] and psychological outcomes [4]. Lately, approaches have moved from purely educational interventions to those that empower patients based on the assumption that they are managers of their own health $[5,6]$. The term patient empowerment refers to a process that enables and facilitates behavior change. The key to empowerment does not necessarily lie in an increased compliance to what the doctor says or prescribes but rather in the opportunity to increase patients' autonomy, and to improve their decision-making capacities [7].

Given the pervasiveness of Information and Communications Technologies (ICT) and their potential, eHealth and mHealth applications have become particularly popular for interventional approaches that aim at empowering patients. Studies in diabetes patients using web-based approaches have indeed shown to effect empowerment levels [8]. With regard to clinical outcomes, web-based interventions were able to reduce blood glucose levels [9] and hospitalization rates [10]. Even though effects found were in general rather small [11], web-based approaches, compared to traditional approaches, yield promising results [12]. Regarding the effectiveness of mobile applications, there is only few solid evidence on diabetes treatment so far, given that the field of mHealth is still very young $[13,14]$. However there are some recent encouraging findings that underscore the potential of mobile use in combination with web-based approaches for diabetes education purposes $[11,15]$.

Most web-based interventions, so far, have not been comprehensively embedded into the healthcare environment surrounding the individual patient. Therefore, more systematical approaches are needed. Personal health records (PHR) that are linked to electronic health records (EHR) have shown to be a promising solution. PHRs allow patients to manage and share their health information using secure pathways with others who are authorized to see them [16]. Particularly in chronic disease management PHRs are of considerable value given that diabetes patients require constant care and regular follow-ups. In addition, time and resource constraints in physicians' everyday practice make PHRs even more useful [17].
Studies that included PHRs and focused on diabetes were able to show that the use of PHRs linked to EHRs would increase for instance the adjustment of diabetes related medication [18] or improve blood glucose levels [19].

Goal-setting is one of the key components facilitating behavior change with the empowerment approach [7]. Goal-setting helps patients to act more independently by providing feedback on their self-management behavior thereby also sustaining motivation and increasing problemsolving skills and self-reflection. Goal-setting does not necessarily have to refer to purely clinical outcomes but also to psychological outcomes such as self-efficacy, which in the long run might influence the continuity or increase of an action set as a goal [20]. Even though goal-setting in collaboration with the treating physician is favorable, only few studies so far were able to show an active involvement of the treating physician. Studies showed that most physicians were reluctant to actively get involved, due to time and resource constraints. The usage of computer-based programs has shown to be helpful to, at least partly, circumvent these issues [20]. However, in spite of physicians' reluctance, most of the interventions, including nondiabetes related studies, that used for example PHRs were mostly physician-oriented and did not take sufficiently the patients' needs into account. For example, patients were only able to access their information via their physicians or treating hospitals. Besides, the general lack of additional information on diabetes treatment or any form of patient support proved those systems to be less attractive to patients [21].

In general, ICT should be used to facilitate communication and information exchange between patient and healthcare providers, which is fundamental to the motivation of the patient. ICT should not rely solely on information provision and data insertion but also contribute to an interactive exchange between the patient and his/ her healthcare providers [22-24].

\section{Objective}

The current study aims at increasing empowerment and self-management behaviors in a sample of diabetes patients type 1 and type 2 in Germany and Turkey by:

- Providing a web-based application, available also for mobile devices, that allows patients to regularly upload their personal health records in form of blood glucose measures or journal entries regarding physical activity, nutrition, etc.

- giving patients the opportunity to systematically define goals and track their improvements over the course of the intervention

- integrating physicians' treatment goals and recommendations into the system 
- systematically integrating the web-based application with electronic and personal health records

\section{Methods/Design}

\section{The web-based application}

The development of the application (EMPOWER system Figure 1) encompasses four main objectives. The first one is to foster self-management with adaptive and secure patient pathways. The pathways are iterative and adaptable to the patients' skills (access, competence and motivation), requirements and needs, which will be assessed by the system according to different maturity levels. Maturity levels - novice, advanced, and expert - refer here to the stage reached by the patient in learning to self-manage his/her diabetes management tasks.

A second objective is to support behavioral changes by integrating actions in patient's daily life through personalized action plans. These actions plans will be based on recommendations from the treating physicians, personalized long-term goals, diabetes-relevant information material, and patients' preferences. In addition, actions can be related to reminders in order to bring them timely into the patient's mind. The third objective is to facilitate selfcontrol by collecting patterns of daily living. Therefore, services for observations of daily living (ODLs) that allow patients to upload vital, physical (e.g. blood glucose levels) and mental parameters and physical and lifestyle activities are included in the system. The last objective is to include an open-source PHR system, which can be integrated into existing PHR or EHR systems. In turn, the EMPOWER system aims at integrating services from these other health applications. Semantic interoperability is supported on the basis of established standards (i.e. ISO/CEN13606 information models).

The EMPOWER system goes beyond already available diabetes self-management applications as it also involves the patients' treating physician. During a routine consultation at the beginning of the intervention phase the

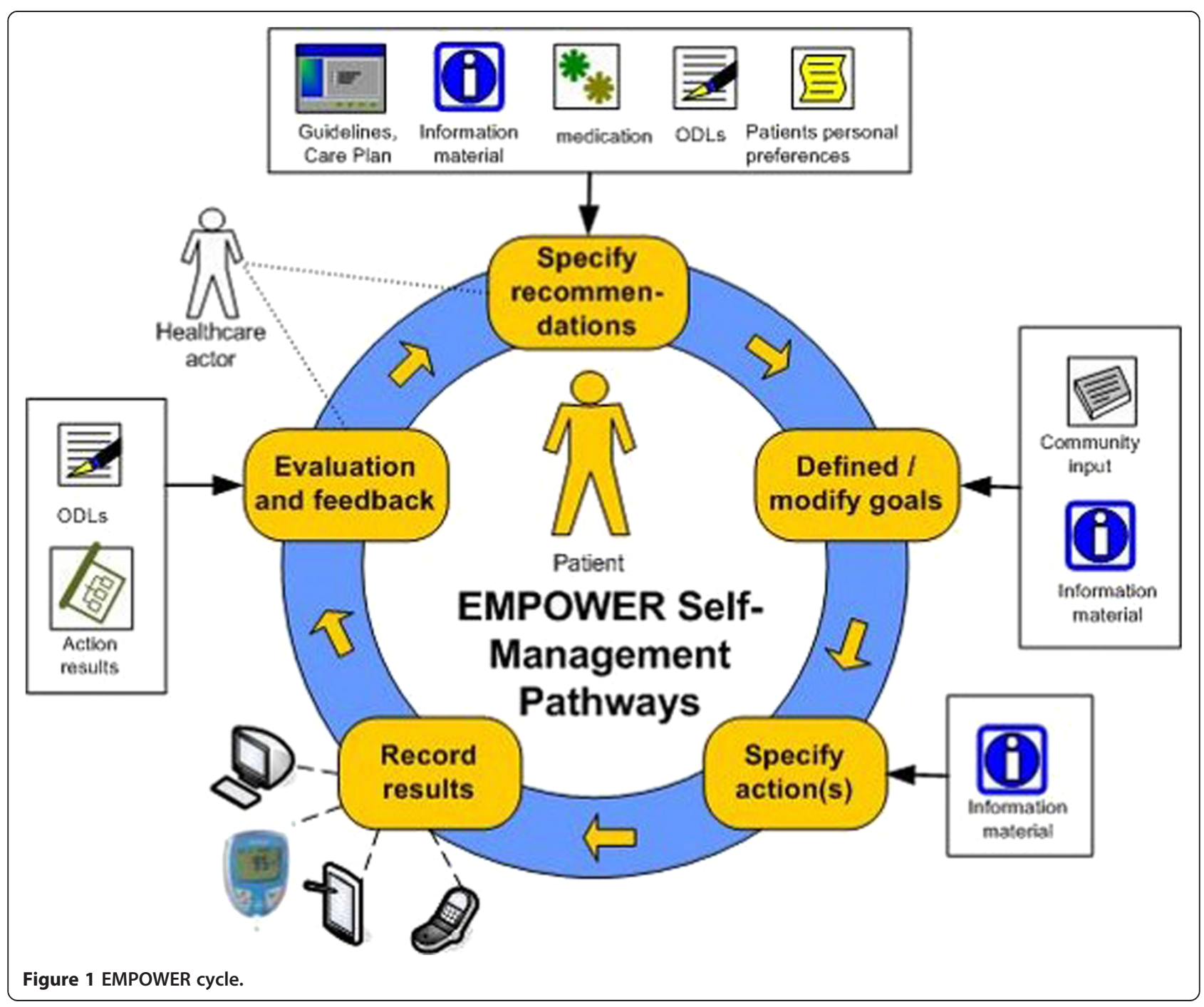


collaborating physicians will specify treatment goals and recommendations regarding self-management goals together with the patient. This goal setting serves as the basis for the use of the EMPOWER system. Based on the patient's consent the treating physician will be able to access the patient's EMPOWER records and follow the patient's self-management activities. Consequently, physicians will be able to detect possible causes for changes in the patient's condition, such as fluctuating blood glucose levels. In addition, the physician will be able to discuss the patient's goal pursuit process and to give advice on diabetes self-management.

From a user experience perspective the EMPOWER system is divided into two main blocks. The first block targeting the treating physician is called recommender engine. The second block is the self-management portal, dedicated to the patients. The physician can monitor the patient's health status through the recommender engine, which receives input from the patient's Observations of Daily Living (ODLs). As a result, the physician can give the patient recommendations. A patient can check his/her physician's recommendations, set his/her personal goals and plan his/her weekly activities in the self-management portal (Figure 2).

The patient can create journal entries during the week in order to get an overview of his/her progress, and use the review at the end of the week to evaluate the journal entries and to plan the activities of the forthcoming week. The patient decides on his/her own whether or not to grant access to his/her data to the physician by using the consent editor.

As a constant support, the patient can also access a section called patient information material, which offers disease related information and can be of help in planning goals and activities. The information material has been written in English for EMPOWER and then translated into German and Turkish. The material is based on existing and acknowledged information sources and educational material for diabetes patients. Since patients suffering from diabetes have different levels of knowledge, the material is designed to address different information needs: from basic information to more detailed, and from generic information to practical advice. For this purpose the Patient Information Material has been structured along three levels of content: Knowledge, Management, and Advice.

\section{Pretest of the EMPOWER Platform}

The pilot application was pretested during a first validation phase in Germany and Turkey. Fifteen diabetes patients, thereof seven from Germany and eight from Turkey, first received a training on how to use the EMPOWER system and then used it for two months. Feedback on usability and usefulness was gathered quantitatively via standardized questionnaires as well as qualitatively through a roundtable discussion. The results of the first validation phase were then used to further develop the system and eliminate existing

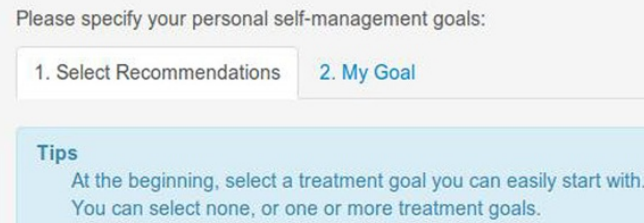

Choose a treatment goal for specifying your own, more detailed self-management goals

\section{My recommendations for self-management from my doctor(s)}

\begin{tabular}{|c|c|c|c|}
\hline$\square$ & Date & Care Provider & Recommendation \\
\hline$\square$ & $\begin{array}{l}\text { Wed Feb } 08 \text { 09:36:00 } \\
\text { CET } 2012\end{array}$ & Hans Schmidt & Take the medications every morning. \\
\hline$\checkmark$ & $\begin{array}{l}\text { Wed Feb 08 09:36:00 } \\
\text { CET } 2012\end{array}$ & Hans Schmidt & Measure and record blood sugar and blood pressure daily. \\
\hline$\square$ & $\begin{array}{l}\text { Wed Feb } 08 \text { 09:36:00 } \\
\text { CET } 2012\end{array}$ & Hans Schmidt & Check your weight on Sunday morning before breakfast. \\
\hline$\square$ & $\begin{array}{l}\text { Wed Feb 08 09:36:00 } \\
\text { CET } 2012\end{array}$ & Hans Schmidt & Change your eating behaviour within the next two months. \\
\hline$\square$ & $\begin{array}{l}\text { Wed Feb } 08 \text { 09:36:00 } \\
\text { CET } 2012\end{array}$ & Hans Schmidt & Do some sports. \\
\hline
\end{tabular}

Figure 2 Self-management portal: Physician's recommendations to the patient. 
difficulties. Besides, the seven patients from Germany also participated in the pretest of the questionnaire on self-management that will be used as a baseline and post-intervention measure to assess the effectiveness of the EMPOWER system.

\section{Study design}

The study is planned as a pre-post-design by administering questionnaires before and after the intervention. The aim is to recruit up to 70 participants at both study sites and then randomly assign them to either the intervention or control group.

After being recruited participants will be asked to give their informed consent. They will then be administered an online questionnaire containing various measures on self-management and socio-demographics to assess their baseline values before using the EMPOWER system. In order to create familiarity with the tool all participants will take part in an introductory training. There, patients will be taught how to download, use, and integrate the application into their daily life.

Participants are supposed to use the system regularly for a period of three months. They will be contacted via telephone on a weekly basis to gather feedback on usability, discuss potential problems concerning the tool and reduce the loss to follow-up. After the intervention has ended patients are again asked to fill in an online questionnaire containing the same measures as the pre-questionnaire plus questions on usability and usefulness of the tool.

\section{Ethical approval}

The project was approved by the Bavarian State Office for Data Protection, Germany and by the Scientific Researches Evaluation Commission of the Ministry of Health of the Turkish Republic.

\section{Recruitment}

It is planned to recruit overall 140 patients. The calculation of 140 participants (70 patients at each study site) is based on a previous study by Schulz and colleagues [25] in the field of web-based interventions. Eligible participants are between 18 and 65 years of age and suffer from diabetes type 1 or 2 . Moreover, they need to have access to internet, own a computer or smart phone and be able to use the EMPOWER application.

In Germany, participants will be recruited via the practice network GOIN in Ingolstadt, Bavaria. The network comprises about 500 medical specialists and general practitioners who care for about 200.000 patients. The administrative staff from the practice network will be in charge of the recruitment of the patients and physicians for the main study. This will be done through personal contact with network members and advertising in practices and the network's magazine.
Turkish participants will be recruited at the Hitit University Endocrinology Clinic of Turkey in Ankara. The Turkish Ministry of Health collaborates with two endocrinologists, two diabetes nurses, and one dietician at the University hospital who will recruit the participants among their patients, and also hired participants for the pretest.

\section{Outcome assessment}

While the pre-questionnaire will only contain questions on self-management related constructs and socio-demographics, the post-questionnaire will also measure the perceived usability and usefulness of the application. Both questionnaires will be administered online and take about 20 to 30 minutes. Pre-postcomparisons will be facilitated by the random and thus anonymous assignment of a unique code for each participant that has to be entered when starting the survey. The questionnaire was created in English and then translated. Where available, already validated translations were used. For those scales that were only available in English, back and forward translations by native speakers of both languages were applied.

\section{Patient information}

Basic recordings about the patients comprise information such as socio-demographic descriptions, number of physician consultations during the intervention phase, or his/her experience with using similar technologies. Besides, the usage of EMPOWER will be recorded for each participant, including the patient's number of logins to the system, the duration of use per visit, or the number of goals entered into the system. The records will show how often and intense the system was actually used by participants, and allow conclusions about the most useful features of the tool. Subjectively reported information from the patients will then be compared to objectively collected data.

\section{Usability}

Usability will be measured using the System Usability Scale by Brooke [26]. The scale was developed to assess the usability of interactive systems and is commonly used for this purpose. It consists of ten items that ask for a subjective assessment of the system, e.g. how easy it was to use it or how well integrated the various features of the system are. Both, reliability and validity of the scale have been proven [27]. Questions to assess the perceived usefulness of the EMPOWER system are based on the Technology Acceptance Model [28,29]. The model considers why technological systems are accepted or rejected by its users and how certain characteristics of the system determine this process. To measure the perceived usefulness of the EMPOWER system 
ten items from Davis' [29] short screening scale on technology acceptance are used and were slightly adapted to the purposes of EMPOWER.

To assess whether the participating physicians perceive the EMPOWER system as useful in fostering patients' self-management semi-structured interviews will be conducted. This will be done during the intervention phase via short telephone interviews to gather current feedback on the tool and more detailed through face-to-face interviews after the intervention.

\section{Empowerment}

Patients' empowerment will be measured using Spreitzer's Empowerment Scale [30] and The Problem Areas in Diabetes (PAID) [31]. Spreitzer's Empowerment Scale consists of twelve statements related to the patient's perceived importance, control, management, and autonomy concerning his/her diabetes. The PAID asks the patient whether he/she perceives twenty aspects, such as being limited concerning nutrition or not having treatment goals, as current problems.

\section{Self-management activities}

The impact of patient empowerment on self-management activities through the use of the EMPOWER system will be assessed by measuring the patients' pre- and postintervention health status, diabetes self-care, health literacy, doctor-patient communication, and empowerment. To assess an overall effect of the application on the health status as perceived by the patient, a self-reported, one item measure will be used. Patients will be asked to rate their health independently from their diabetes to avoid negatively biased answers due to the chronic condition. Diabetes self-care will also be measured subjectively, using the SDSCA as developed by Toobert and Glasgow [32] and revised by Toobert and colleagues [33]. The scale focuses on the patients' self-care across various dimensions such as diet, exercise, blood sugar testing, foot care, and smoking. The questions refer to the last seven days of a participant's routine diabetes self-care and therefore represent a comprehensive insight into the patient's selfmanagement that will be completed by the objective data stemming from the user tracking.

\section{Health literacy}

As an adequate level of heath literacy is paramount for being an effective self-manager [34] possible changes in health literacy due to the use of the EMPOWER system are assessed. Therefore, the Newest Vital Sign (NVS) [35], an objective test of functional health literacy in form of a nutrition label will be employed. Further, a subjective measure consisting of three items related to the perceived ability to understand written medical information, namely the Chew Items [36,37], will be used.
In order to gain insight in the patients' knowledge specifically about diabetes a range of knowledge items will be asked. Doctor-patient communication will be measured with scales from Kaplan [38] and Heisler [39]. While the first one assesses a physician's decision making style concerning the patient's involvement in treatment decisions, the latter one focuses on the provider communication. It thus considers the patient's satisfaction with his/her doctor's communication concerning disease and treatment.

\section{Data analysis}

Data will be analyzed using the statistics software package SPSS, version 21, from IBM. To detect possible changes and their predictors' descriptive statistics and regression analyses will be applied. In order to compare pre- and post-results as well as control and intervention group dependent and independent t-tests will be calculated.

\section{Discussion}

The interplay between multiple sources, including EHRs, patients' own registered information and treating physicians' recommendations on one platform can have important implications for general practice. By actively integrating these features in one comprehensive platform the EMPOWER system may not only improve self-management activities in diabetes patients but will also facilitate physician's work by having all important information readily available on one platform. In addition, the system may help improve the relationship between doctor and patient and foster a more systematic discussion with the patient on further disease management strategies and potential intervention points.

\section{Limitations}

Limitations are prone to occur with regard to patient recruitment. The intervention will have to foresee potential dropouts due to the length of the intervention and potential research overload on the side of the patient.

In addition, physicians are asked to be actively involved in the program but time constraints and resource limitations are likely to impact their involvement. Nevertheless, with regard to patient retention rates physicians are of crucial importance in order to promote and sustain the intervention.

Lastly, given the fairly short amount of time of three months, behavioral changes may not be easily detectable, therefore regular use of the EMPOWER system has to be made sure in form of reminders and regular updates regarding changes of the system.

\section{Abbreviations}

EHR: Electronic health records; ICT: Information and communication technologies; ODL: Observations of daily living; PHR: Personal health records. 


\section{Competing interests}

The authors declare that they have no competing interests.

\section{Authors' contributions}

SM, MF, RL participated in the conceptualization and the design of the study. SM, MF, RL, PJS drafted and revised the final manuscript. All authors read and approved the final manuscript.

\section{Authors' information}

MF is a postdoctoral research fellow at the Institute of Communication and Health.

$\mathrm{RL}$ is a PhD candidate at the Institute of Communication and Health. $\mathrm{SM}$ is a PhD candidate at the Institute of Communication and Health. PJS is full professor and director of the Institute of Communication and Health.

\section{Acknowledgements}

The EU funds this study in the scope of the 7th Framework Programme, Theme ICT (Project identifier: FP7-ICT-2011-288209).

\section{Received: 27 October 2014 Accepted: 2 March 2015}

Published online: 20 March 2015

\section{References}

1. Chen L, Magliano DJ, Zimmet PZ. The worldwide epidemiology of type 2 diabetes mellitus - present and future perspectives. Nat Rev Endocrinol. 2011;8(4):228-36.

2. Dagogo-Jack S. Preventing diabetes-related morbidity and mortality in the primary care setting. J Natl Med Assoc. 2002;94(7):549.

3. Norris SL, Lau J, Smith SJ, Schmid CH, Engelgau MM. Self-Management education for adults with type 2 Diabetes A meta-analysis of the effect on glycemic control. Diabetes Care. 2002;25(7):1159-71.

4. Steed L, Cooke D, Newman S. A systematic review of psychosocial outcomes following education, self-management and psychological interventions in diabetes mellitus. Patient Educ Couns. 2003;51(1):5-15.

5. Funnell MM, Anderson RM. Patient empowerment: a look back, a look ahead. Diabetes Educ. 2003;29(3):454-64.

6. Funnell MM, Brown TL, Childs BP, Haas LB, Hosey GM, Jensen B, et al. National standards for diabetes self-management education. Diabetes Care. 2011;34(Sup. 1):S89-96.

7. Anderson RM, Funnell MM. Patient empowerment: myths and misconceptions. Patient Educ Couns. 2010;79(3):277-82

8. Samoocha D, Bruinvels DJ, Elbers NA, Anema JR, van der Beek AJ. Effectiveness of web-based interventions on patient empowerment: a systematic review and meta-analysis. J Med Internet Res. 2010;12(2):e23.

9. Costa BM, Fitzgerald KJ, Jones KM, Dunning T. Effectiveness of IT-based diabetes management interventions: a review of the literature. BMC Fam Pract. 2009;10:72

10. Dorr DA, Wilcox AB, Brunker CP, Burdon RE, Donnelly SM. The effect of technology-supported, multidisease care management on the mortality and hospitalization of seniors. J Am Geriatr Soc. 2008:56(12):2195-202.

11. Pal K, Eastwood SV, Michie S, Farmer A, Barnard ML, Peacock R, et al Computer-based interventions to improve self-management in adults with type 2 diabetes: a systematic review and meta-analysis. Diabetes Care. 2014;37(6):1759-66.

12. Ramadas A, Quek KF, Chan CKY, Oldenburg B. Web-based interventions for the management of type 2 diabetes mellitus: a systematic review of recent evidence. Int J Med Inform. 2011;80(6):389-405.

13. Fiordelli M, Diviani N, Schulz PJ. Mapping mHealth research: a decade of evolution. J Med Internet Res. 2013;15(5):e95.

14. Free C, Phillips G, Galli L, Watson L, Felix L, Edwards P, et al. The effectiveness of mobile-health technology-based health behaviour change or disease management interventions for health care consumers: a systematic review. PLOS Med. 2013;10(1):e1001362.

15. Saffari M, Ghanizadeh G, Koenig HG. Health education via mobile text messaging for glycemic control in adults with type 2 diabetes: a systematic review and meta-analysis. Prim Care Diabetes. 2014:8(4):275-85.

16. Tang PC, Ash JS, Bates DW, Overhage JM, Sands DZ. Personal health records: definitions, benefits, and strategies for overcoming barriers to adoption. Patient Educ Couns. 2006;13(2):121-6.
17. Tenforde M, Jain A, Hickner J. The value of personal health records for chronic disease management: what do we know? Fam Med. 2011;43(5):351-4.

18. Grant RW, Wald JS, Schnipper JL, Gandhi TK, Poon EG, Orav EJ, et al. Practice-linked online personal health records for type 2 diabetes mellitus: a randomized controlled trial. Arch Intern Med. 2008;168(16):1776-82.

19. Tang PC, Overhage JM, Chan AS, Brown NL, Aghighi B, Entwistle MP, et al. Online disease management of diabetes: Engaging and Motivating Patients Online With Enhanced Resources-Diabetes (EMPOWER-D), a randomized controlled trial. J Am Med Inform Assoc. 2013;20(3):526-34.

20. Bodenheimer T, Handley MA. Goal-setting for behavior change in primary care: an exploration and status report. Patient Educ Couns. 2009;76(2):174-80.

21. Archer N, Fevrier-Thomas U, Lokker C, McKibbon KA, Straus SE. Personal health records: a scoping review. J Am Med Inform Assoc. 2011;18(4):515-22.

22. Glasgow RE, Kurz D, King D, Dickman JM, Faber AJ, Halterman E, et al. Twelve-month outcomes of an Internet-based diabetes self-management support program. Patient Educ Couns. 2012;87(1):81-92.

23. Boren SA, Gunlock TL, Peeples MM, Krishna S. Computerized learning technologies for diabetes: a systematic review. J Diabetes Sci Technol. 2008;2(1):139-46.

24. Piette JD. Interactive behavior change technology to support diabetes self-management where do we stand? Diabetes Care. 2007;30(10):2425-32.

25. Schulz PJ, Rubinelli S, Caiata Zufferey M, Hartung U. Coping with chronic lower back pain: designing and testing the online tool ONESELF. J Comput Mediat Commun. 2010;15(4):625-45

26. Brooke J. SUS: A "quick and dirty" usability scale. In: Jordan PW, Thomas B, McClelland IL, Weerdmeester B, editors. Usability Evaluation in Industry. London: Taylor and Francis; 1996. p. 189-94.

27. Lewis JR, Sauro J. The factor structure of the system usability scale. First International Conference, Human Centered Design 2009, San Diego, CA, USA, July 19-24, Proceedings. 94-103.

28. Davis F. Perceived usefulness, perceived ease of use, and user acceptance of information technology. MIS Quart. 1989;13(3):319-40.

29. Davis F. User acceptance of information technology: System characteristics, user perceptions and behavioural impacts. Int J Man Mach Stud. 1993;38:475-87.

30. Spreitzer GM. Psychological empowerment in the workplace: Dimensions, measurement, and validation. Acad Manage J. 1995:38(5):1442-65.

31. Welch GW, Jacobson AM, Polonsky WH. The problem areas in diabetes scale: an evaluation of its clinical utility. Diabetes Care. 1997;20(5):760-6.

32. Toobert DJ, Glasgow RE. Assessing diabetes self-management: the summary ofdiabetes self-care activities questionnaire. In: Bradley C, editor. Handbook of Psychology and Diabetes. Chur: HarwoodAcademic; 1994. p. 351-75.

33. Toobert DJ, Hampson SE, Glasgow AR. The summary of diabetes self-care activities measure: results from 7 studies and a revised scale. Diabetes Care. 2000;23(7):943-50

34. Schulz PJ, Nakamoto K. Health literacy and patient empowerment in health communication: The importance of separating conjoined twins. Patient Educ Couns. 2013:90(1):4-11.

35. Weiss BD, Mays MZ, Martz W, Castro KM, DeWalt DA, Pignone MP, et al. Quick assessment of literacy in primary care: the newest vital sign. Ann Fam Med. 2005;3(6):514-22.

36. Chew LD, Bradley KA, Boyko EJ. Brief questions to identify patients with inadequate health literacy. Fam Med. 2004;36(8):588-94.

37. Chew LD, Grill JP, Snyder A, Bradley KA, Nugent SM, Baines AD. Validation of screening questions for limited health literacy in a large VA outpatient population. J Gen Intern Med. 2008;23(5):561-6.

38. Kaplan SH, Greenfield S, Ware Jr JE. Assessing the effects of physician-patient interactions on the outcomes of chronic disease. Med Care. 1989:27(3):S110-27.

39. Heisler M, Bouknight RR, Hayward RA, Smith DM, Kerr EA. The relative importance of physician communication, participatory decision making, and patient understanding in diabetes self-management. J Gen Intern Med. 2002;17(4):243-52 